\title{
Experimental Study on a Model of Bulk Wind Energy Collecting System
}

\author{
Zhongjian Sun \& Zhibo Tang (Corresponding author) \\ School of Naval Architecture and Civil Engineering, Zhejiang Ocean University \\ Wenhua Road 109, Dinghai District, Zhoushan, Zhejiang Province, China \\ E-mail: sunzhongjian@hotmail.com, Zhibo_tang@yahoo.com
}

Received: November 21, 2010

Accepted: December 17, 2010

doi:10.5539/mas.v5n2p143

The research is financed by Science and Technology Department of Zhejiang Province, Science Technology Bureau Zhoushan City Zhejiang Province, Zhejiang Ocean University, and Zhejiang Ship Advanced Manufacturing Technology Research and Development Center.

\begin{abstract}
In this paper, we propose a vertical axis wind energy collecting system and bulk wind energy collecting system. This system may be integrated with the urban high buildings, and produce the electricity by the means of collecting high-altitude wind energy. We design a model and do series of experiments on the model and get some results. Results show that power output reaches a maximum when the angle is $45^{\circ}$, turbine starts to work at the wind speed of $1.8 \mathrm{~m} / \mathrm{s}$. We find that it has a strong ability to adapt to random direction and low speed wind.
\end{abstract}

Keywords: Bulk wind energy collecting system, Wind energy generator, Experimental study, Output efficiency

\section{Introduction}

Stable, clean and efficient renewable energy sources are caused more and more attention to world. Today fossil fuels consisting of coal, oil, and natural gas are finite resources and will be completely exhausted in next decades. (Mathew, 2006)

Wind energy now represents a significant part of the electricity generation in a number of European countries. This phenomenon is not only limited to European countries. The important of wind energy capacities is also being discussed and planned in rapid developing countries such as China, India or Brazil. Wind energy is one of the most attractive renewable energy options in terms of both cost competitiveness and environmental impacts. (Sundqvist and Soderholm, 2003,pp.3-41)The quantum of energy associated with the wind is enormous. With today's technology, wind is an environment friendly and economically viable source of energy, which can be tapped into a commercial scale. Increasing the value of wind generation through improving the performance of collecting systems is one of the priorities in wind energy research for the coming years.(Giebel and Brownsword et al., 2003; Thor and Weis-Taylor, 2002, pp.73-75)

In recent years, the main development of wind turbine is still with horizontal axis. Horizontal axis wind turbines (HAWT) have their axis of rotation horizontal to the ground and almost parallel to the wind stream (Figure 1). Horizontal axis machines have some distinct advantages such as low cut-in wind speed and easy furling.(Mathew, 2006) However, the traditional methods of energy generation has its inherent flaws, mainly involved the following points: 1) Utilization efficiency of wind energy (the collecting efficiency) is very low, the wind utilization efficiency in the range of effective cross-section is usually only $17 \%$; 2) Changes of wind direction seriously affect the efficiency of wind energy collecting; 3 ) The only way to increase wind energy output is to enhance the size of blades, which causes shorter fatigue life and lower structural stability. Long length blade also causes larger costs of maintenance. 4) It needs a lot of ground.

The rotation axis of vertical axis wind turbine (VAWT) is vertical to the ground and almost perpendicular to the wind direction such as the Darrieus wind turbine, the Savonius wind turbine and the Musgrove turbine. (Mathew, 2006)The vertical axis wind turbine has advantages that can work in any wind direction with speed controlling unit; it can be placed on the ground, and so on. Its development is restricted by long axis and low conversion efficiency. The main product in wind energy market is multi-stage variable-speed gearbox wind-driven energy unit. The main mode of large wind energy unit is variable-speed, dual-fed, variable pitch and constant frequency. Now there are direct-drive energy unit with small components, which can reduce the loss of transmission chain 
and downtime. It also has the low costs of maintenance and makes a growing share of market.

As we know, the wind resource is very rich on the islands, mountains, high altitude and sea. (Xu and $\mathrm{He}$ et al., 2010, pp.4439-4444)This provides an opportunity for us to use the wind energy. It also gives us the inspiration of invention. Do we have the possibility to use rich wind resources at high-altitude in city? Can we put new wind energy turbine on the top of high-rise buildings to solve the own electricity supply and even to provide electricity to others? The significance of the new wind energy generation system has the answer to these questions. The turbine design is a compromise which involves limiting capital cost and at the same time extracting the maximum energy from the average wind conditions. (Grimble, 1996, pp.143-161)The new wind energy system that we design considers the amount of energy. The core component of the new wind system is named -- "bulk wind energy collecting system."

\section{Structure of Bulk Wind Energy Collecting System}

The bulk wind energy collecting system (also known as bulk wind turbines) are made up of five main parts, which are blades, baffle, rotational axis, supporting frames and covers. Through the bulk wind turbine, wind energy may be changed into other forms of energy. Bulk wind energy collecting system is intentionally designed to set up at the top of modern high-rise buildings in city. An integration of high-rise building and wind energy collection system comes true. (i.e. Figure 2 and Figure 3). Five main parts of the system will be introduced as following.

\subsection{Blades}

Wind turbine blade (Figure 2) designed and plays a key role on the wind energy collecting efficiency. When wind blows over the blades, the blades drive the rotational axis, then start the generators. The blade design concerns the number, shape, size and materials of blades. Integration of bulk wind turbine and building leads to a huge size of wind turbine equipment, which needs big size of vertical blades with high strength. The space truss structure has been applied in the blade design to enhance its strength. Meanwhile, we need to reduce the weight of blades and the structure non-homogeneity as much as possible.

\subsection{Baffle}

Design of baffle (Figure 2 and Figure 3 ) is a new concept in the bulk wind turbine system. The role of the baffle is to guide the wind direction, turning irregular direction wind into fixed direction wind for blades. The baffle can not only improve the efficiency of energy conversion, but also maintain the better stability of the wind energy. Our intention is to install a reinforced concrete baffle at the top of high-rise building. The baffle can maintain strength and stiffness. The baffle can also make wind turbine be integrated with building.

\subsection{Rotational Axis}

The rotational axis (Figure 2) connects the generator and blades. When the blades are turning, the generator is also turned up. The friction coefficient of the rotational axis must be reduced. So we generally design the axis into the form with rolling friction. The center of rotational axis is connected with the column of the building to improve the overall stability of the wind turbine when it is set up at the top of the high-rise building.

\subsection{Cover and Supporting Frames}

Cover and supporting frames (Figure 4 and Figure 5) are designed to maintain the stability of wind turbine model. At the top of high-building, we may remove the supporting frames, because the central axis and the baffles have been directly integrated to the building, which can give enough strength and stiffness.

\subsection{Generator}

The wind energy generator is the basic device that transforms wind energy into electrical energy. Coil cuts magnetic line in the magnetic field and then transforms the mechanical energy into electrical energy. In the experiment, we have used DC Motor to facilitate the measurement of energy.

\section{Experimental Study of Bulk Wind Energy Collecting System}

We design and manufacture a small scale bulk energy collecting system model (Figure 4 and Figure 5) and carry out the experiment of energy output in the laboratory.

\subsection{Introduction of Model}

Our model design starts from Solidworks 2010 (3d mechanical design software). According to the literature of other researchers, we finally determined the sizes of blade and baffle. The size of blade is $456 \mathrm{~mm} * 97 \mathrm{~mm}$ and $470 \mathrm{~mm} * 50 \mathrm{~mm}$ for baffle. Shapes of blades and baffles are both rectangle. The main material of the model is of ABS (Acrylonitrile -butadiene-styrene). The rotation axis, bolts and nuts are made with steel. Through the 
experiments, it turns out that the strength of ABS is enough for our experiment. The bulk wind collecting system may be easily disassembled. Baffles are connected with a circle by linkages (Figure 5). We place a central circle to regulate the positions of baffles, and baffles are placed uniformly along the circumference. Angle between baffle and blade radius direction can be adjusted from 15 degrees to 90 degrees. The supporting frames are designed to fix the system and facilitate the installation of generator.

We use SFG-type low-noise fan (power $3 \mathrm{~kW}$, frequency $50 \mathrm{HZ}$, speed $1450 \mathrm{r} / \mathrm{min}$, voltage $380 \mathrm{~V}$ ) to blow the blades. This fan can't justify the wind speed. We just regulate speed blowing to the system by adjusting the distance between fans and turbine. Fluke F112 digital multi-meter is used to detect current and voltage. We can get the output energy from current and voltage. The rotary blade propeller anemometer is used to test the wind speed. The 45ZYN004 DC motor (Voltage of $24 \mathrm{~V}$, current of 1.4A, speed $1700 \mathrm{r} / \mathrm{min}$ ) is the generator.

Totally five groups of students are coordinated in the experiments. One group manages the fan to control the wind direction and speed. One takes care of DC motor. One operates digital multi-meter and reads the values of output current and voltage. One measures the wind speed field near the model. And the last one records all of the data.

\subsection{Process of Experiment}

Next we proceed to describe the details of experiments, which may be divided in two steps.

Firstly, we set up 8 pieces of blades and 16 pieces of baffles and hold wind speed at a constant $7.2 \mathrm{~m} / \mathrm{s}$. The angle between blades and baffles are changed from $20^{\circ}$ to $80^{\circ}$. We have found that, when the angle between blades and baffles comes to be $45^{\circ}$, energy output reaches a maximum (

Table 1). Next we attempt to change the numbers of blades and baffles. The results show that this change does not influence the output efficiency significantly, as long as the number of blades holds more than 4 pieces, and the baffles more than 12 .

In second step, we keep the number of blades, 8 pieces and baffles 16 , and the angle between baffles and blades is $45^{\circ}$. We adjust the fan to acquire different wind speed, and then measure the values of wind energy outputs.

\subsection{Results and Discussion}

According to the study from a large number of experiments, we obtain that the bulk wind turbine output energy varies with different wind speed. Table 2 has collected the results we have measured with a relatively accurate test data. And this table describes the conversion efficiency of the wind energy collection model.

Then we get the relationship curve between output power and corresponding wind speed (Figure 6). The $\mathrm{x}$-axis denotes the wind speed and the y-axis presents output energy. The units are meter/second and watt, respectively.

We find that the turbine starts to work at the wind speed of $1.8 \mathrm{~m} / \mathrm{s}$ (very low speed), i.e., wind-grade level 2 , which is the main advantage of our system. And we have estimated that the wind utilization efficiency is about $29 \%$ or more, which is much better than that of traditional wind energy collecting system, with average $17 \%$ as mentioned in the section of introduction.

It should be noted that, in our experiments, when the wind speed is higher than $12 \mathrm{~m} / \mathrm{s}$, the wind power output does not increase significantly. Possibly, this is because of the limitations of our simple model, and we need more experiments to resolve it.

To increase the energy output, we may widen the blades and/or make the model higher (i.e. longer blades). This will be quite important issue. We need to make more models with different sizes for future studies. Furthermore, we need to carry out out-door experiments, with condition of natural wind, to confirm our current results.

\section{Conclusion}

A new wind energy collecting system has been designed and tested through many experiments. Experiment results show that the angle between blade and baffle must be $45^{\circ}$, which is the best angle for wind energy collecting. The system may start to work with low wind speed-- $1.8 \mathrm{~m} / \mathrm{s}$. And wind energy utilization rate may reach to $29 \%$, which shows obvious advantage compared with traditional wind energy collecting system. The numbers of blades and baffles needed for the system have been determined through various experiments. And eventually experiments are carried out systematically to estimate the relationship between wind energy output and wind speed, then to measure the wind energy collecting efficiency of our system.

\section{References}

Giebel, G. and R. Brownsword, et al. (2003). The state-of-the-art in short-term prediction of wind power. A literature overview. Risoe National Laboratory. 
Grimble, M. (1996). Horizontal axis wind turbine control: Comparison of classical, LQG andH $\infty$ designs. Dynamics and Control 6 (2): 143-161.

Mathew, S. (2006). Wind Energy - Fundamentals, Resource Analysis and Economics, Springer Berlin Heidelberg.

Sundqvist, T. and P. Soderholm (2003). Valuing the environmental impacts of electricity generation: A critical survey. RESEARCH REPORT-INTERNATIONAL INSTITUTE FOR APPLIED SYSTEMS ANALYSIS IIASA RR VIII (2): 3-41.

Thor, S. E. and P. Weis-Taylor (2002). Long-term research and development needs for wind energy for the time frame 2000-2020. Wind Energy 5 (1): 73-75.

$\mathrm{Xu}, \mathrm{J}$. and D. He, et al. (2010). Status and prospects of Chinese wind energy. Energy 35 (11): 4439-4444.

Table 1. System output power of angles of blades and baffles at wind speed $14.2 \mathrm{~m} / \mathrm{s}$

\begin{tabular}{|c|c|c|c|c|c|}
\hline angle $\left(^{\circ}\right)$ & $20^{\circ}$ & $30^{\circ}$ & $45^{\circ}$ & $60^{\circ}$ & $80^{\circ}$ \\
\hline output power $(\mathrm{W})$ & 1.92 & 3.84 & 4.59 & 3.93 & 1.32 \\
\hline
\end{tabular}

Table 2. System output power at different wind speed

\begin{tabular}{|c|c|c|c|}
\hline Wind speed (m/s) & current (A) & Voltage (V) & output power (W) \\
\hline 1.8 & 0 & 0 & 0 \\
\hline 3.6 & 0.058 & 0.868 & 0.05 \\
\hline 5.6 & 0.112 & 1.288 & 0.14 \\
\hline 6.6 & 0.205 & 1.656 & 0.34 \\
\hline 7.2 & 0.235 & 2.224 & 0.52 \\
\hline 8.6 & 0.283 & 3.222 & 0.91 \\
\hline 9.6 & 0.415 & 3.802 & 1.58 \\
\hline 10.8 & 0.506 & 5.012 & 2.54 \\
\hline 11.5 & 0.555 & 5.548 & 3.08 \\
\hline 12.5 & 0.689 & 6.267 & 4.32 \\
\hline 14.2 & 0.728 & 6.302 & 4.59 \\
\hline
\end{tabular}

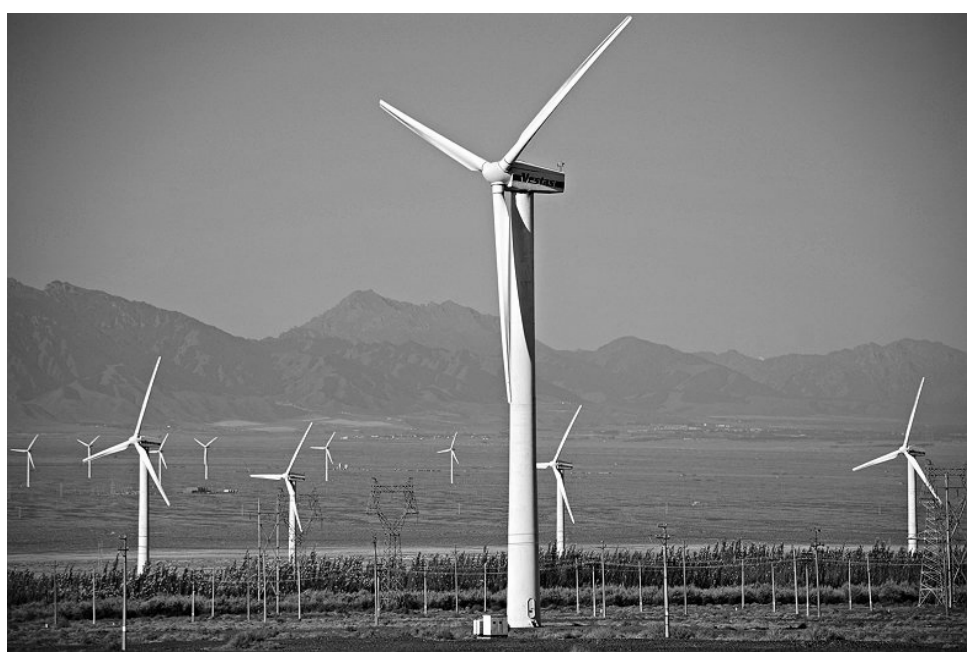

Figure 1. Three bladed horizontal axis wind turbines (World Wind Power Net, 2010) 


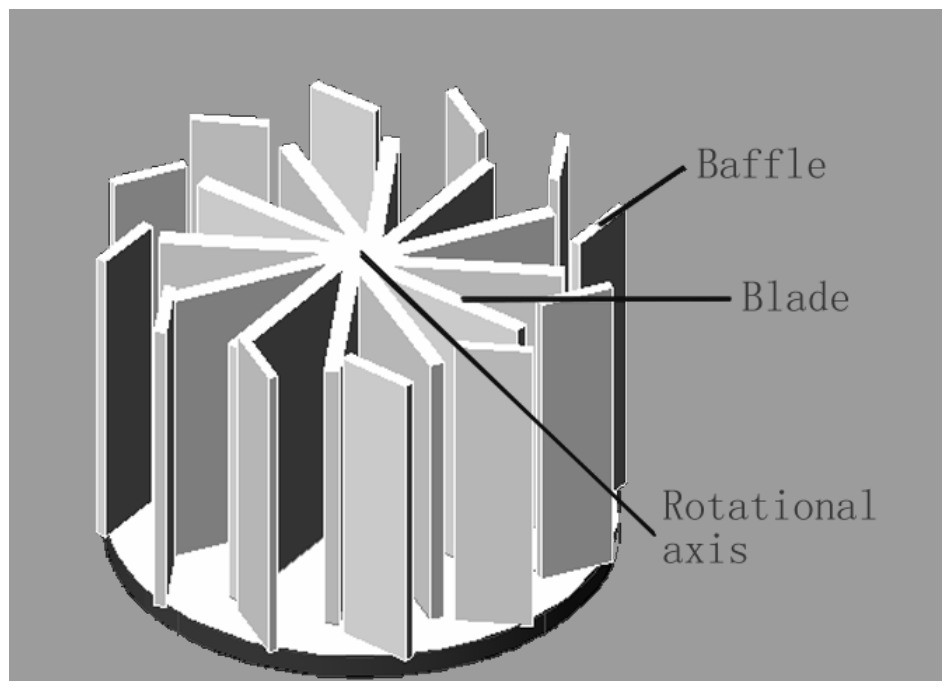

Figure 2. Bulk wind energy collecting system three-dimensional section view

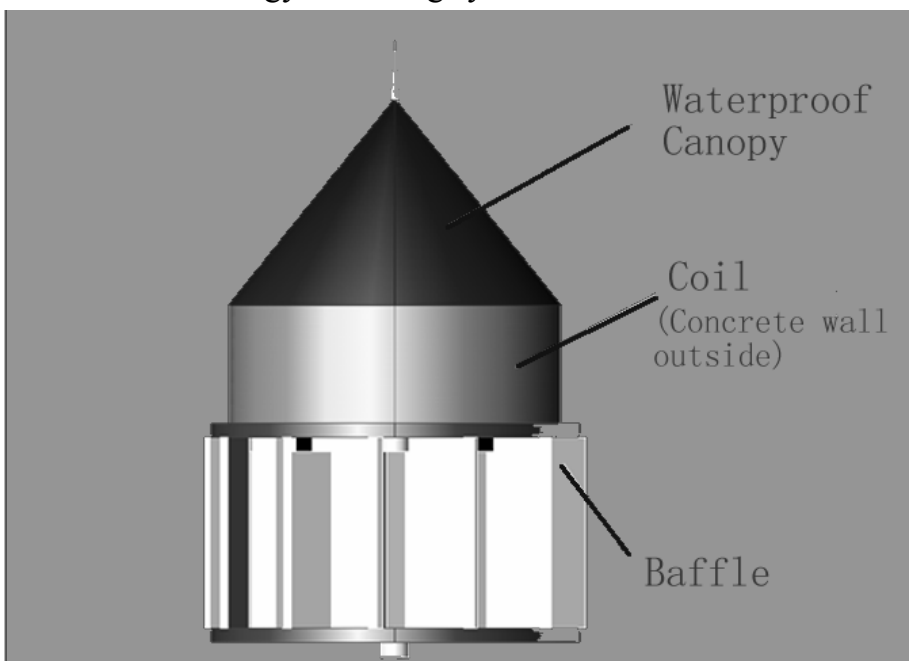

Figure 3. Elevation view of bulk wind energy collecting system (high-altitude wind energy capture)

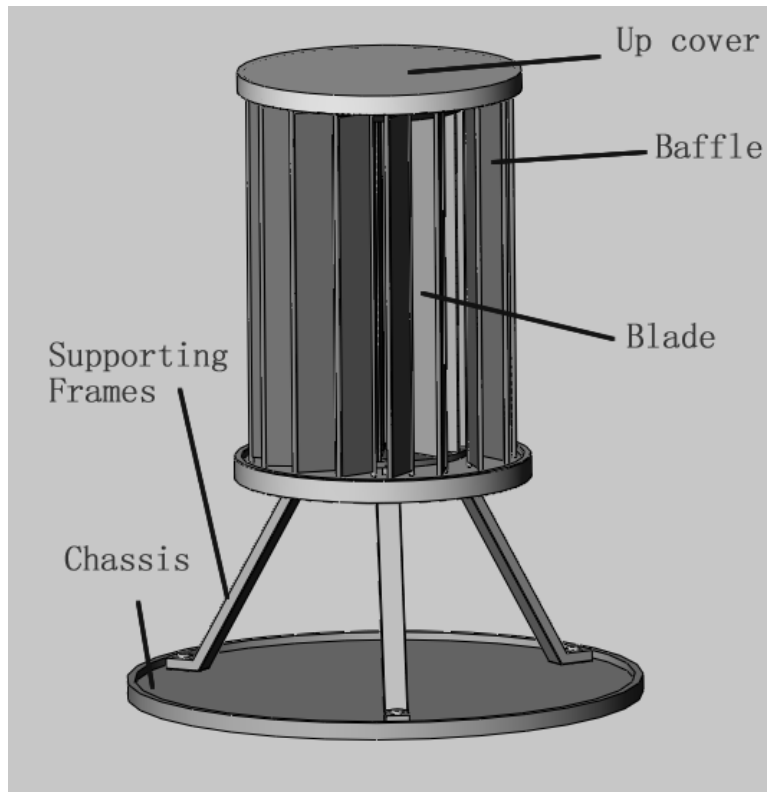

Figure 4. 3D view of the model 


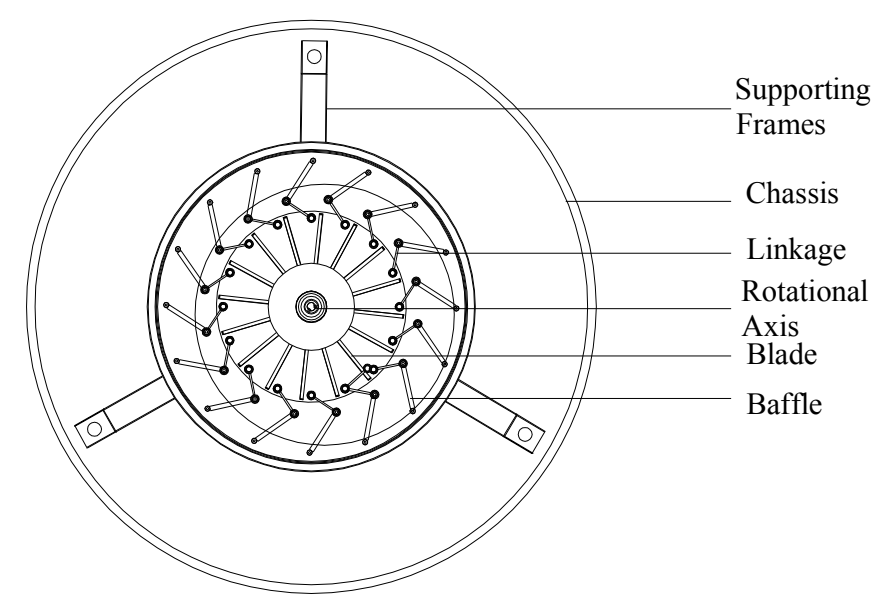

Figure 5. Sectional view of the model

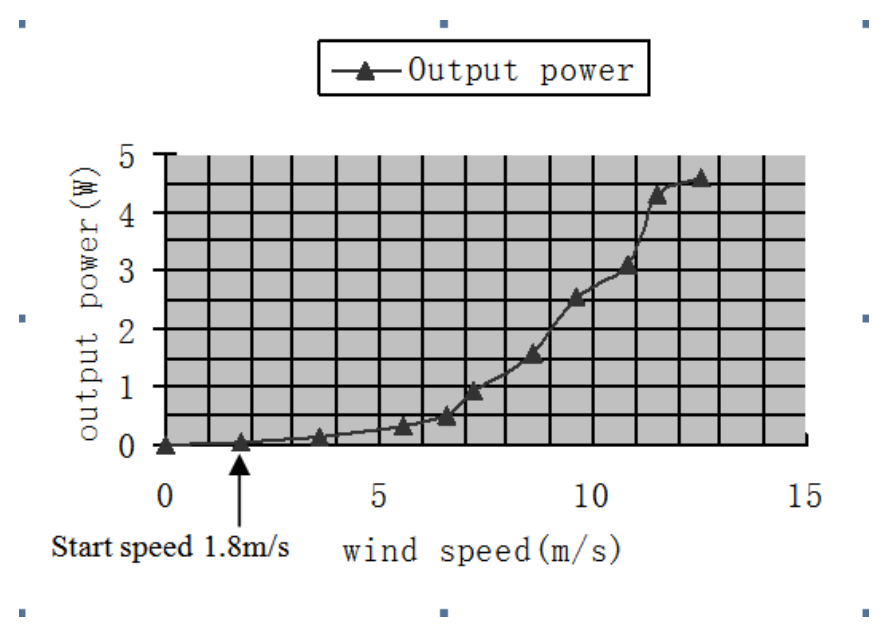

Figure 6. Relationship curve between output energy of bulk wind energy collecting system and wind speed 